\title{
High-efficiency and multi-frequency polarization converters based on graphene metasurface with twisting double L-shaped unit structure array
}

\author{
Ming Chen ${ }^{1,2, *, \#, X i a o f e i} X^{2}{ }^{2, *}$, Linzi Chang ${ }^{2}$,Congyun $\mathrm{Wang}^{2}$, and Deping Zhao ${ }^{2}$ \\ ${ }^{1}$ Guangxi Key Laboratory of Precision Navigation Technology and Application, Guilin \\ University of Electronic Technology, Guilin 541004, Guangxi, China \\ ${ }^{2}$ Guangxi Experiment Center of Information Science, Guilin 541004, Guangxi, China \\ ${ }^{\#}$ Corresponding author: mchenqq2011@163.com \\ *These authors contributed equally to this work
}

\begin{abstract}
In this work, a high-efficiency and tunable dual-frequency reflective polarization converter composed of graphene metasurface with twisting double L-shaped unit is firstly realized. Numerical results demonstrate that the device can convert a linearly polarized wave to its cross-polarized wave, and meantime it can also convert to a circularly polarized wave. Subsequently, one thickness of $500 \mathrm{~nm} \mathrm{SiO} 2$ layer sandwiched by two graphene metasurfaces with similar pattern is stacked on the top of the two-layered structure, a four-frequency efficient reflective polarization converters is realized. Above all, those working frequencies can also be dynamically tuned within a large frequency range by adjusting the Fermi energy of the graphene, without reoptimizing and refabricating the nanostructures, which paves a novel way toward developing a controllable polarization converter for mid-infrared applications.
\end{abstract}

Keywords: Graphene; Metasurface; Multi-frequency; Polarizer; Mid-infrared 


\section{Introduction}

Efficiently manipulating light is a hot research area by controlling the state of optical polarization in many optical applications. It is a conventional method to manipulate the polarization of electromagnetic (EM) waves using optical gratings and optical activity crystals, or utilizing Faraday effects and birefringence effects materials [1, 2]. In recent years, metamaterial $(\mathrm{MM})$ have opened new routes towards the efficient manipulation of EM polarization due to its exotic properties, such as negative refraction [3,4], invisible cloaking [5] and super-lenses [6]. Tons of MM structures have been used for implementing polarization conversion, such as metallic nanoparticles [7,8], metallic nanoslots [9-11] and three-dimensional chiral MMs [12-14]. Many basic MM structures, such as nanoparticle or nanoslot, exhabit the high-efficiency of polarization, on account of it can excite localized surface plasmon $[9,10]$. However, those polarization converters are uncontrolled. It is practically inconvenient because the polarimetric devices can work at different working frequencies only by changing the structure of geometries or the substrates. Therefore, graphene as an adjustable material has been paid considerable attention.

Graphene, an attractive two-dimensional (2D) carbon material, shows unique optical properties. A variety of mid-infrared components based on graphene plasmonic structure have been demonstrated in the past decade, including perfect absorbers [15-17], filters $[18,19]$, and modulators [20,21]. In addition to these devices, polarization converters [22-24], as a device to control polarization state in communication and spectroscopy, are often needed. Hua Cheng et al. proposed single-frequency tunable mid-infrared polarization converters employing L-shaped graphene MM [22]. Gang Yao et al. reported a polarization rotator device based on graphene MM [23]. These proposed polarization converters can convert linearly polarized (LP) light to its orthogonal polarized light and a wide frequency tunability. However, the conversion efficiency of the aforementioned 
works are about $80 \%$. Therefore, it is highly urgent to fabricate multi-frequency and high-efficiency polarization converters.

In this letter, we firstly present an active plasmonic cross-reflective polarization converter (CPC) based on graphene metasurface (GMS), which is capable of working at two resonant frequencies with high-efficiency. The numerical results show that the conversion efficiency reaches $99.45 \%$ at $25.46 \mathrm{THz}$ and $99.77 \%$ at $34.86 \mathrm{THz}$ under normal-incident LP waves. On the basis of above-mentioned structures, a bi-layered GMS is proposed to enhance its interaction effect dramatically. Theoretical results show that the bi-layered GMS can achieve cross-polarization with high efficiency for two orthogonal LP waves in four individual separated frequency, where LP waves can be mostly converted to its cross-polarization. Two types of GMS CPCs can be dynamically regulated in a large frequency range by adjusting the Fermi energy of the graphene. The physical mechanisms are elucidated by taking advantage of the induced surface current distributions. The geometrical form of the twisting double L-shaped structure unit of the GMS is very regular and it can be fabricated easily.

\section{Theoretical model and design}

The schematic diagrams of two types of GMS CPCs are sketched Fig. 1. Fig. 1(a) depicts a single-layer GMS CPC, which consists of twisting double L-shaped unit GMS on the top layer, and a $\mathrm{SiO}_{2}$ layer backed by a thick gold ground plane to totally suppress the wave transmission. The ground plane is made of a $100 \mathrm{~nm}$ thick gold, and the thickness of dielectric layer is $h_{1}=1200 \mathrm{~nm}$. One unit cell of the array structure shows in the upper right-hand corner, the geometric parameters are as follows: $\Lambda=125 \mathrm{~nm}, L=30$ nm, $w=30$ nm. Fig. 1(b) describes a multi-frequency GMS CPC. It is composed of two layers GMS with the same twisting double L-shaped and bottom layer with gold, but the size of upper layer graphene is a little smaller than the below one. The geometric parameters are detailedly described in the caption. 

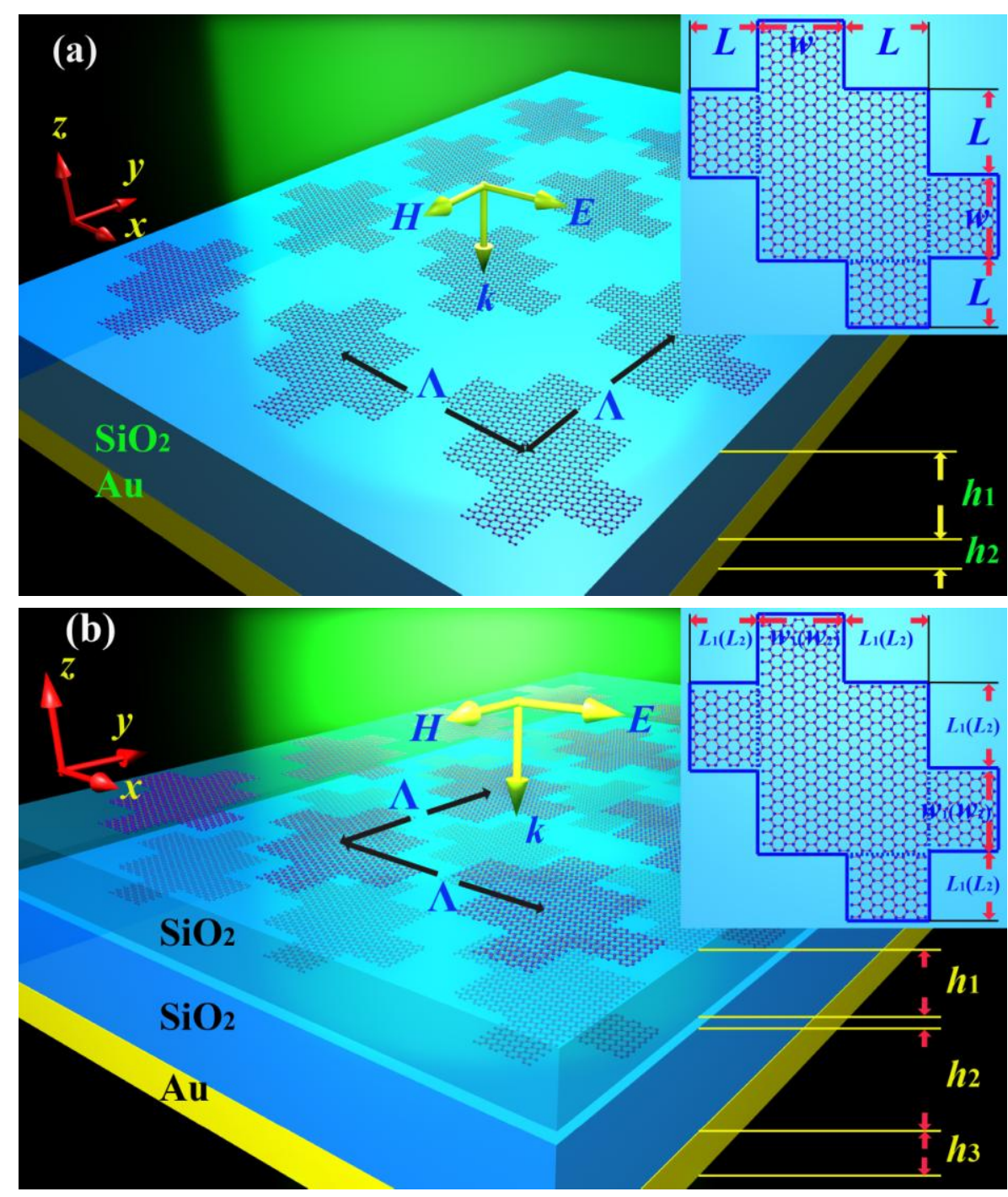

Fig. 1. (a) Schematic diagram of the single-layer GMS CPC and the geometric parameters of unit cell are: $\Lambda=125 \mathrm{~nm}, L=30 \mathrm{~nm}, w=30 \mathrm{~nm}$, respectively. $h_{1}=1200 \mathrm{~nm}$ is for $\mathrm{SiO}_{2}$ layer and $h_{2}=100 \mathrm{~nm}$ is for Au layer. (b) Schematic model of the bi-layer MGS CPC, the unit dimensions of up-side GMS are: $L_{1}=30 \mathrm{~nm}, w_{1}=30 \mathrm{~nm}$, and the unit dimensions of GMS, sandwiched by two $\mathrm{SiO}_{2}$ layers with the thickness $h_{1}=500 \mathrm{~nm}$ and $h_{2}=1200 \mathrm{~nm}$, are $L_{2}=30 \mathrm{~nm}, w_{2}=25 \mathrm{~nm}$, respectively. $h_{3}=100 \mathrm{~nm}$ is for Au layer. The period $\Lambda$ of the two GMSs is equal and $\Lambda=130 \mathrm{~nm}$.

The gold permittivity in the infrared spectral regime is described by using the Drude model with the plasma frequency $\omega_{p}=1.37 \times 10^{16} s^{-1}$ and the damping coefficient 
$\gamma=4.08 \times 10^{13} s^{-1}$. Without considering the magnetostatic bias field and spatial dispersion, the surface conductivity of graphene $\sigma(\omega)$ can be acquired by Kubo formula [25] :

$$
\begin{gathered}
\sigma(\omega)=\sigma^{\text {intra }}(\omega)+\sigma^{\text {inter }}(\omega) \\
\sigma^{\text {intra }}(\omega)=\frac{2 \kappa_{B} e^{2} T}{\pi \hbar^{2}} \ln \left[2 \cosh \left(\frac{E_{f}}{2 \kappa_{B} T}\right)\right] \frac{i}{\omega+i \tau^{-1}} \\
\sigma^{\text {i net }}(\omega)=\frac{e^{2}}{4 \hbar}\left[H\left(\frac{\omega}{2}\right)+\frac{4 i \omega}{\pi} \int_{0}^{\infty} \frac{H(\varepsilon)-H(\omega / 2)}{\omega^{2}-4 \varepsilon^{2}} d \varepsilon\right] \\
H(\varepsilon)=\frac{\sinh \left[\hbar \varepsilon /\left(\kappa_{B} T\right)\right]}{\cosh \left[E_{f} /\left(\kappa_{B} T\right)\right]+\cosh \left[\hbar \varepsilon /\left(\kappa_{B} T\right)\right]}
\end{gathered}
$$

where $e, \hbar$ and $\kappa_{B}$ representing the charge of an electron, the reduced Planck's constant and the Boltzmann constant, respectively. $T$ is the absolute temperature which is fixed at $300 \mathrm{~K}$, we suppose momentum relaxation time $\tau=2 \mathrm{ps}$. The equivalent permittivity of graphene can be derived from $\varepsilon=1+\frac{j \sigma}{\varepsilon_{0} \omega t}$ [26], where $t$ is the thickness of graphene sheet. The surface conductivity material model specified by Eq. (1)-(4) is applied in both single and multiple graphene layers [27].

To numerically investigate the performance of our design, considering the illumination of a normally incident EM wave and propagating along the of $-z$ direction. The structure is simulated using the frequency domain solver of CST Microwave Studio 2015, which is based on the finite integral method. The periodic boundary of unit cell are applied in all $x-z$ and $y-z$ planes, and open for the $z$-direction in the environment of free space. Owing to the geometry is symmetrical, the simulation results of $y$-polarized light incidence is same to $x$-polarized light incidence. Consequently, we only calculate the complex reflection coefficients under an $x$-polarized incidence. Assuming $\theta$ is the angle between its polarization direction and the $x$ axis, then the electric field of the wave is given by $E_{0}(\cos \theta, \sin \theta)^{T} \exp (-i \omega t)$, among $E_{0}$ is the incident wave amplitude. The 
relationship between the incident filed and the complex-valued reflected electric fields $\left(E_{r}\right)$ is as follows:

$$
E_{r}\left(\begin{array}{c}
\cos \varphi \\
\sin \varphi
\end{array}\right)=E_{0}\left(\begin{array}{ll}
R_{x x} & R_{x y} \\
R_{y x} & R_{y y}
\end{array}\right)\left(\begin{array}{c}
\cos \theta \\
\sin \theta
\end{array}\right)
$$

where $R_{x x}$ is the co-polarization reflection coefficients of $x$-polarized waves ( $x$-to- $x$ ) and $R_{y x}$ is the cross-polarization reflection coefficients ( $x$-to-y), as well as $R_{y y}$ and $R_{x y}, \phi$ is the time-variable polarization angle of the real part of reflected wave. The subscripts $x$ and $y$ indicate the polarization state of EM waves. Then, polarization conversion ratio (PCR) is defined as $P C R=\left|R_{y x}\right|^{2} /\left[\left|R_{x x}\right|^{2}+\left|R_{y x}\right|^{2}\right][5,28]$, here, the part of absorption is not considered. Absorptivity $A(\omega)$ is obtained from $A(\omega)=1-R(\omega)-T(\omega)$, where $R(\omega)$ and $T(\omega)$ are the total reflectivity and transmissivity as functions of frequency $\omega$, respectively. The transmissivity $T(\omega)$ is zero because the metal film is used as ground plane, which results in $A(\omega)=1-R(\omega)$. Defining the phase difference of $R_{x x}$ and $R_{y x}$ as $\Delta \varphi_{y x}=\arg \left(R_{y x}\right)-\arg \left(R_{x x}\right) ; \Delta \varphi_{y x}$ can take value range within $[-\pi, \pi]$, which includes all possible polarization states (linear, circular, elliptic) in the reflected wave can be achieved.

\section{Results of analysis and Discussions}

The dual-frequency CPC-the function of both line-to-line and line-to-circle-has been realized. Its absorptivity $(A(\omega))$, cross-polarization $\left(R_{y x}\right)$ and co-polarization $\left(R_{x x}\right)$ reflection coefficient as a function of frequency for the proposed structure is shown in Fig. 2(a), where the Fermi energy of graphene is applied at $1.0 \mathrm{eV}$. We can observe two overlapped reflection peaks in $R_{y x}$ at $25.46 \mathrm{THz}$ and $34.86 \mathrm{THz}$, and the corresponding dips in $R_{x x}$ with low reflections. These features of $R_{y x}$ and $R_{x x}$ indicate a function of cross 
polarization conversion in the reflection mode. Besides, the absorptivity keeps at a low level relatively. The strongest absorption occurs at resonance frequencies, due to the interaction between electric and magnetic fields becomes stronger at the resonance frequency. The relationship between PCR and reflection phase difference is plotted in Fig. 2(b). The polarization state and the revolving direction are decided by the phase difference [29]. It can be clearly seen that the PCR has two maximum values as $99.45 \%$ at $25.46 \mathrm{THz}$ and $99.77 \%$ at $34.86 \mathrm{THz}$, respectively. At that two frequencies, nearly all energies of the $x$-polarized incident wave are converted to the $y$-polarized reflected one, and the phase differences between $R_{y x}$ and $R_{x x}$ come close to $0^{\circ}$ and $180^{\circ}$, respectively. In addition, the calculated value of phase difference are $88.1^{\circ}, 91.7^{\circ}$, and $92.1^{\circ}$ in Fig. 2(b) at $26.06 \mathrm{THz}, 33.92 \mathrm{THz}$, and $35.61 \mathrm{THz}$, respectively. When the phase differences are equal to $90^{\circ}$ and $R_{y x}=R_{x x}$, it deduces the pure circular polarization wave. At these three frequencies, the $R_{y x}=R_{x x}$ with $0.68,0.68$, and 0.67 , respectively, it indicates that LP wave converts to $\mathrm{CP}$ wave. Compared with the graphene-based CPC proposed by Hua Cheng [22], there are two cross polarization convert frequencies and highly convert efficiency in the dual-frequency CPC by us. Thus, our research is valuable.
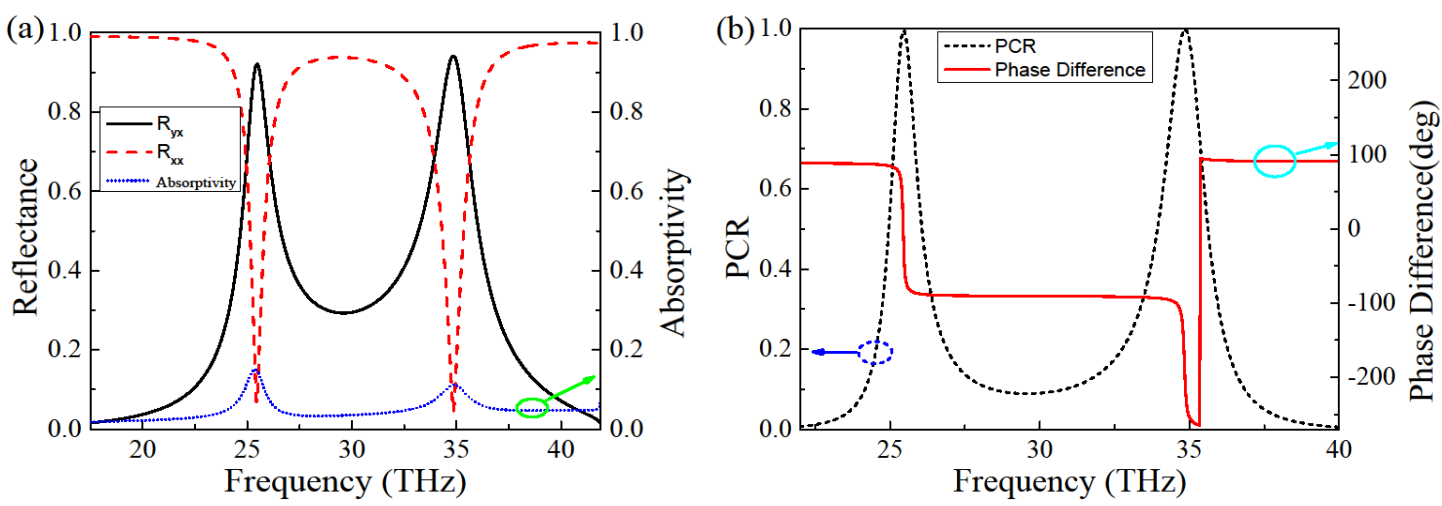

Fig. 2. (a) Simulated reflection spectra of designed polarizer under normal incidence (b) PCR and phase difference of reflectance for single-layer CPC.

To get an insight into the two polarization conversion frequencies of the polarization converter, the surface current distributions between top layer surface and metallic ground 
at two plasmon resonance frequencies are presented. Fig. 3(a) shows the induced surface current distributions of the front and back layers at resonance frequency of $25.46 \mathrm{THz}$. The electric field $\boldsymbol{E}$ is along the $x$-axis, and the induced magnetic field $\boldsymbol{H}$ is along the up-left direction which is decomposed into $x$-direction $\left(\boldsymbol{H}_{x}\right)$ and $y$-direction $\left(\boldsymbol{H}_{y}\right)$. The $y$ component $\boldsymbol{H}_{y}$ of induced magnetic field $\boldsymbol{H}$ is shown in Fig. 3(a). The components of induced magnetic field perpendicular to the incident electric field $\boldsymbol{E}$ cannot occur at the cross-couple because of having the same direction with incident magnetic field. On the other hand, the components of induced magnetic field parallel to the incident electric field $\boldsymbol{E}$ can induce an electronic field (induced electronic field) perpendicular to the incident electric field $\boldsymbol{E}$, and the induced electronic field results in the polarization conversion. The same mechanism is applied at $34.86 \mathrm{THz}$, as shown in Fig. 3(b). From Fig. 3(a) and (b), we observe the intensity of surface current at $34.86 \mathrm{THz}$ is a bit stronger than that at $25.46 \mathrm{THz}$, which shows a stronger cross-couple between graphene and bottom layer metal at $34.86 \mathrm{THz}$.
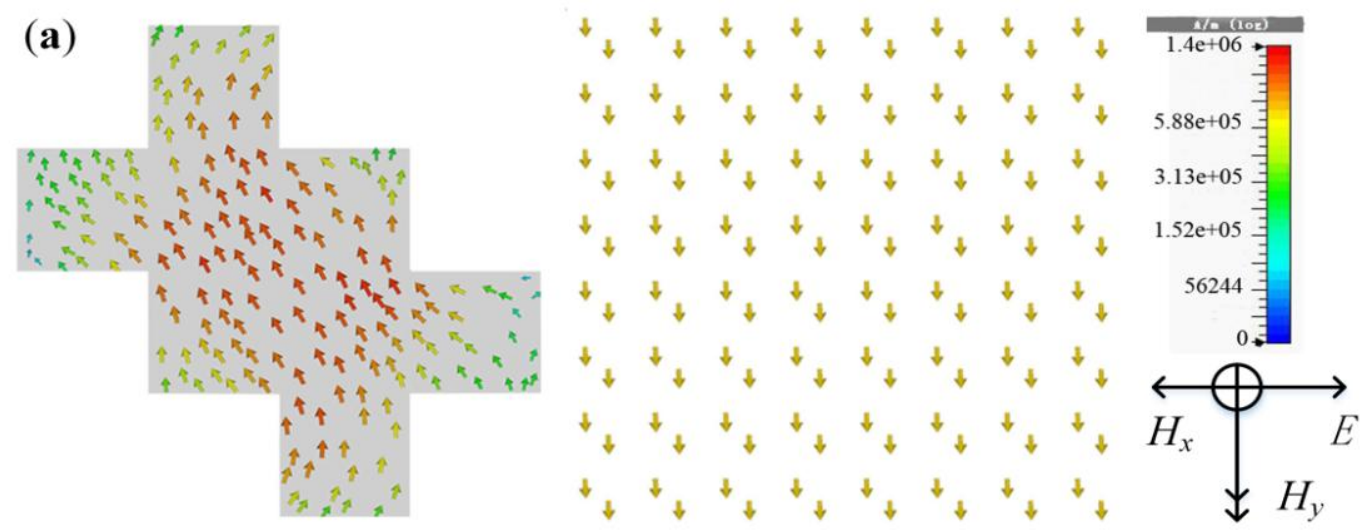



Fig. 3. Surface current distributions of the L-shaped CPC: the front layer (left) and the bottom metallic layer (right) at the resonant frequencies for incident $x$-polarized waves (a) at $25.46 \mathrm{THz}$ and (b) at $34.86 \mathrm{THz}$.

For the purpose of achieving optimum polarization rotation effect, we perform different geometric dimension studies by varying the structure size. First, we keep other parameters unchanged, and increase the period of unit cell from $125 \mathrm{~nm}$ to $185 \mathrm{~nm}$ in a step of $20 \mathrm{~nm}$. Subsequently, the incident angle $\theta$ ranges from $0^{\circ}$ to $60^{\circ}$ with the gradient of $20^{\circ}$. The corresponding PCRs for these two cases are plotted in Fig. 4(a) and (b), respectively.
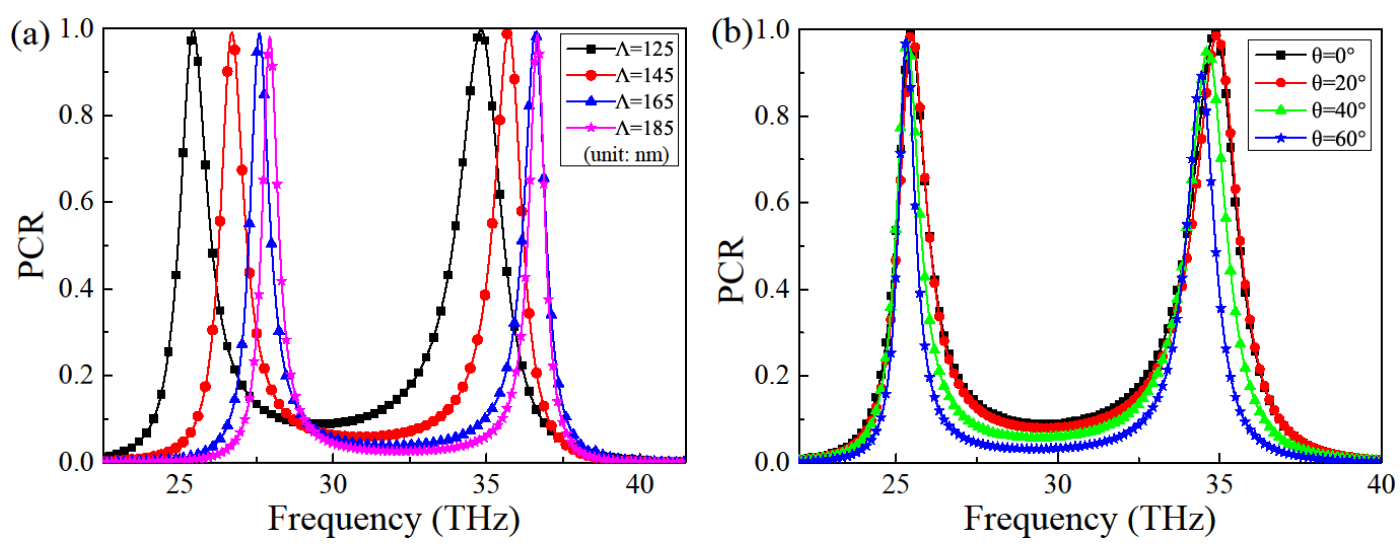

Fig. 4. (a) PCR of different GMS period and (b) PCR of different incident angles.

It is seen from Fig. 4(a) that for each value of $\Lambda$, there are two excited localized surface plasmon modes and the strongest mode is excited with $\Lambda=125 \mathrm{~nm}$. Two frequency 
modes undergoes a blue shift. In Fig. 4(b), it is clearly seen that the high PCR is maintained over the angle range from $0^{\circ}$ to $60^{\circ}$, but it can keep a relatively stable performance. The underlying reason is the higher oblique incidence weakens the reciprocity between EM wave and graphene. In addition, it is obvious that the PCR of high-frequency mode decline faster than the low-frequency one, because the high-frequency mode is more sensitive with the variation of incident angles.

The tunability of frequency is the most highlight characteristics of CPC based on graphene in practical application. The Femi energy of graphene can be tuned from about $-1 \mathrm{eV}$ to $1 \mathrm{eV}$ by chemical doping or electrical gating [30] and it has been used in experiments [31,32], which suggests a great potential for tunable devices. Figure 5(a) shows the PCRs of our proposed device for different graphene Fermi energies. As the graphene Fermi energy increases from $0.7 \mathrm{eV}$ to $1.0 \mathrm{eV}$, the PCR is tunable over a wide range of frequencies, and the enhancement of PCR has been achieved. It is seen that the resonant frequency occur a blue shift obviously. The resonance condition can account for the blue shift of resonant frequency. The wave vector of surface plasmons along the GMS satisfies the equation $k_{s p p}=\hbar \omega_{r}^{2} / 2 \alpha_{0} E_{f} c$ [33], where $\alpha_{0}=e^{2} / \hbar c=1 / 137$ is the fixed structure constant [34]. The resonant frequency can be written as $f_{r}=\sqrt{\alpha_{0} E_{f} / 2 \pi^{2} \hbar c L_{s}} \propto \sqrt{E_{f} / L_{s}}$ [35], among $L_{s}$ represents the resonance related structure parameter. Thus, the simulated result tallies with the theoretical calculations.

The relaxation time of graphene could affect the performance of our proposed polarization converter, which is expressed as $\tau=\mu E_{f} / e V_{f}^{2}$ among $\mu$ and $V_{f}$ are the carrier mobility and the Fermi velocity(about $10^{6} \mathrm{~m} / \mathrm{s}$ ), respectively. Fig. 5(b) plots the PCRs for various relaxation time while other parameters keep invariability. As the value of $\tau$ increases, the PCRs upgrade with increased peak values. This upgrading of PCR with increasing relaxation time is due to the decreased free-carrier loss in graphene, which enhance the plasmonic oscillation of GMS. While the charge carriers reach its threshold 
value, the PCR will remain invariable. Therefore, a good quality of graphene sheet with relatively larger relaxation time is beneficial to obtaining a high efficiency polarization converter.


Fig. 5. (a) PCR under different Fermi energy of graphene film. (b) PCR for $\tau=0.25 \mathrm{ps}, 0.5 \mathrm{ps}, 0.75 \mathrm{ps}$, $1.0 \mathrm{ps}$ and $2.0 \mathrm{ps}$.

The multi-layer structure has been adopted to improve the performance of device and it shows good results [36,37]. Consequently, a bi-layer GMS polarization converter with four resonance frequency is proposed. Two layers GMS and gold are divided by $\mathrm{SiO}_{2}$ spacer with thickness $h_{1}=500 \mathrm{~nm}$ and $h_{2}=1200$ respectively, the period of structure with $\Lambda=130 \mathrm{~nm}$, the other geometrical parameters of the twisting double L-shaped unit structure, as shown in Fig. 1(b). Fig. 6 shows the PCR of bi-layer polarization converter. It is readily observed that four significant resonance peaks are corresponding to near perfect polarization at $21.30 \mathrm{THz}, 26.17 \mathrm{THz}, 28.57 \mathrm{THz}$ and $34.98 \mathrm{THz}$. This middle wide bandwidth results from a superposition of two localized surface plasmon frequencies arising from the coupling of the two layer GMS. 


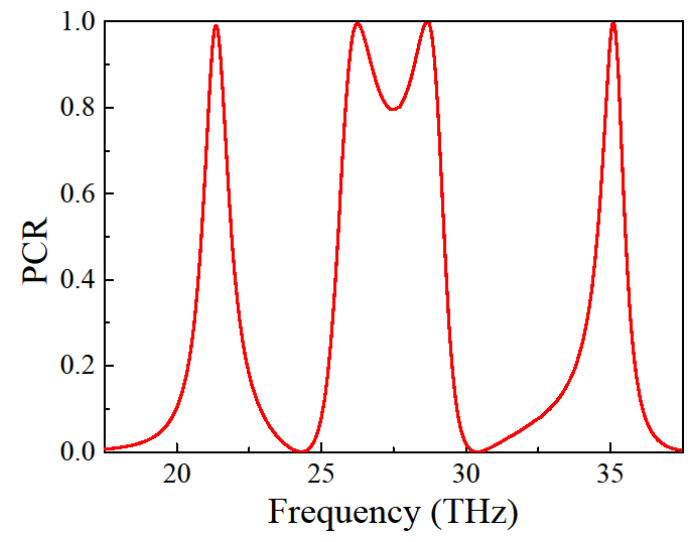

Fig. 6. Calculated PCR of proposed four-frequency polarizer.

To study the relationship between the interval $h_{1}$ and bi-layer CPC, we now only change it from 300 to $900 \mathrm{~nm}$ with an increment of $200 \mathrm{~nm}$. Fig.7(a) indicates the PCRs of our polarization converter for different $h_{1}$, which confirms the optimal $h_{1}$ selected as $500 \mathrm{~nm}$. The reason is that the resonance coupling of between upper and bottom metasurface exist an optimum distance, hence, the increase or reduction of its distance will lessen near-field coupling efficiency. It is found that the resonance frequency comes up shift because the perfect coupling is achieved difficultly.

Furthermore, the polarization spectrum of bi-layer CPC for different Fermi energy has been simulated, as shown in Fig.7(b). It is clearly seen that the higher PCRs with increasing Fermi energy and the resonant frequencies shift upward. It is noteworthy that the PCR can be adjusted in broad band of frequencies when the Fermi level is varied from $0.8 \mathrm{eV}$ to $1.0 \mathrm{eV}$. This tuning property makes graphene-based CPCs more attractive than the metal-based ones, since the tuning is realized by controlling the Fermi energy without adjusting the geometric parameters. 

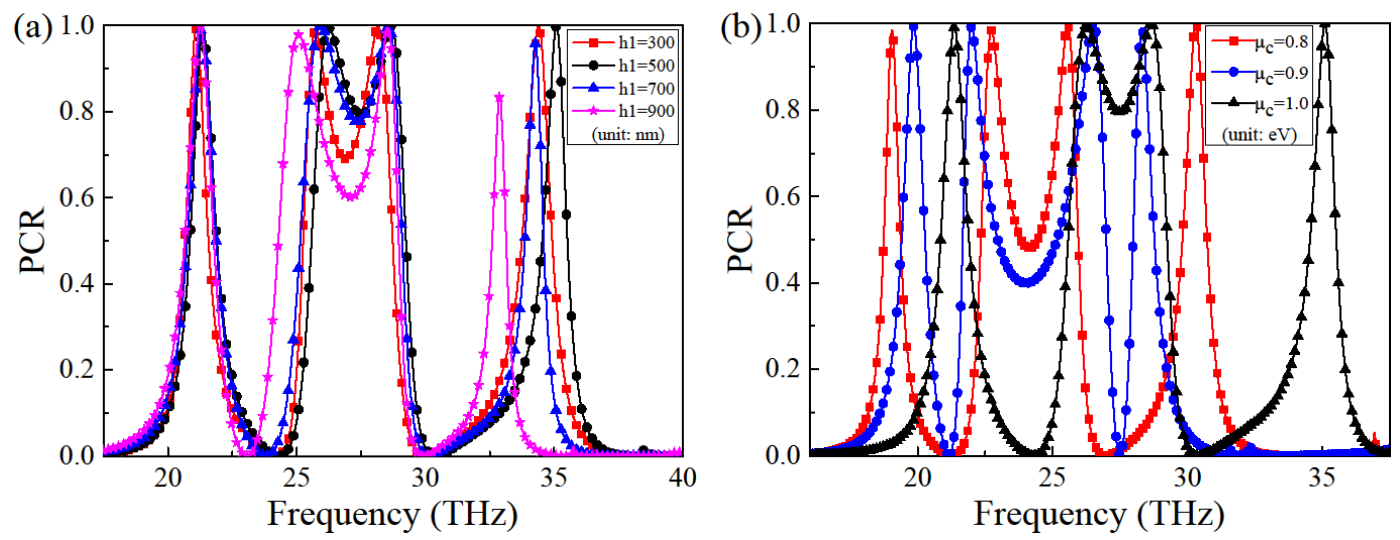

Fig. 7. (a) The polarization spectra for diverse interval and (b) polarization spectra under different Fermi energy.

Finally, we investigate the angular dependence of bi-layer CPC. The PCRs for an incident angle $\theta$ ranging from $0^{\circ}$ to $60^{\circ}$, as shown in Fig. 8. It is observed that the perfect polarization begins to fall obviously when oblique incidence is $60^{\circ}$, but the cross polarization features is still maintained. The less angular dependence of PCR in our device is related to the much smaller feature size of graphene.

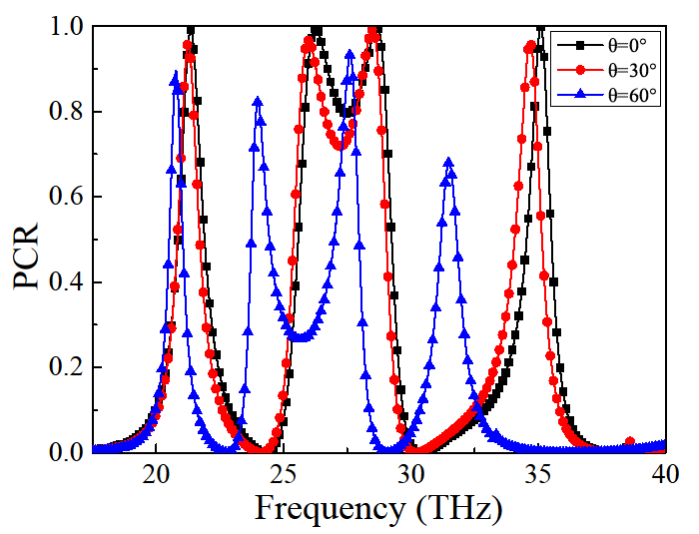

Fig. 8. Polarization spectrum under diverse oblique incidence angles.

\section{Conclusion}

In conclusion, we have proposed two types of cross-polarization converters based on GMS in mid-infrared regime. One type of proposed single-layer GMS with twisting double L-shaped CPC can convert a linearly polarized wave to its cross polarized wave at two different resonant frequencies, which conversion efficiency $(99.45 \%$ at $25.46 \mathrm{THz}$ 
and $99.77 \%$ at $34.86 \mathrm{THz}$ ) is high. This device can also be used as a LP-to-CP converter at $26.06 \mathrm{THz}, 33.92 \mathrm{THz}$, and $35.61 \mathrm{THz}$. The other type with two layer GMS is a four-frequency cross-polarization converter, which can simultaneously transform LP light to the cross-polarization counterpart at $21.30 \mathrm{THz}, 26.25 \mathrm{THz}, 28.65 \mathrm{THz}$ and $35.10 \mathrm{THz}$ with conversion efficiency $99.11 \%, 99.47 \%, 99.68 \%$ and $99.60 \%$, respectively. The resonant frequencies and output polarization states can be dynamically tuned by only adjusting the Fermi energy, without reoptimizing and refabricating the structures. These cross-polarization converters show some potential applications in communication field and integrated optical systems.

\section{Acknowledgments}

The authors thank the National Science Foundation Council of China under Contract No. 61640409, the research fund from Guangxi Key Laboratory of Precision Navigation Technology and Application, Guilin University of Electronic Technology, under Contract No.DH201506, for their support. 


\section{References}

[1] S. Mallick, M. Miteva, L. Nikolova, J. Opt. Soc. Am. B. 14 (5) (1997) 1179-1186.

[2] D. Irvine-Halliday, M. R. Khan, P. G. Zhang, Opt. Engineering. 39 (5) (2000) 1310-1315.

[3] S. C. Jiang, X. Xiong, Y. S. Hu, et al., Phys. Rev. X. 4 (2) (2014) 021026.

[4] X. Huang, D. Yang, H. Yang, J. Appl. Phys. 115 (10) (2014) 103505.

[5] J. Ding, B. Arigong, H. Ren, et al., Opt. Express. 22 (23) (2014) 29143-51.

[6] R. Li, Z. Guo, W. Wang, et al., Plasmonics. 10 (5) (2015) 1167-1172.

[7] C. Helgert, E. Pshenay-Severin, et al., Nano. Lett. 11 (10) (2011) 4400-4.

[8] M. Beruete, M. Navarro-Cía, et al., J. Appl. Phys. 103 (5) (2008) 1-3.

[9] C. Qin, B. Wang, H. Huang, et al., Opt. Express. 22 (21) (2014) 25324-32.

[10] S. Ke, B. Wang, H. Huang, et al., Opt. Express. 23 (7) (2015) 8888-900.

[11] G. Yao, F. Ling, J. Yue, et al., Opt. Express. 24 (2) (2016) 001518.

[12] M. Danaeifar, N. Granpayeh, et al., Appl. Opt. 52 (22) (2013) 68-72.

[13] C. Huang, Y. Feng, et al., Physical Review B. 85 (19) (2012) 1614-1621.

[14] L. Wu, M. Zhang, B. Zhu, et al., Appl. Phys. B. 117(2) (2014) 527-531.

[15] S. Thongrattanasiri, F. H. L. Koppens, et al., Phys. Rev. Lett. 108(4) (2012) 047401 .

[16] B. Vasić, R. Gajic, et al., Appl. Phys. Lett. 103(26), (2013) 261111.

[17] Z. Fang, Y. Wang, A. E. Schlather, et al., Nano Lett. 14(1) (2014) 299-304.

[18] K. Yang, S. Liu, S. Arezoomandan, et al., Appl. Phys. Lett. 105(9) (2014) 093105.

[19] H. J. Li, L. L. Wang, et al., Appl. Phys. Express. 7 (2) (2014) 343-352.

[20]Z. Li and N. Yu, et al., Appl. Phys. Lett. 102(13) (2013) 131108 .

[21] Y. Yao, R. Shankar, M. A. Kats, et al., Nano Lett. 14(11) (2014) 6526-6532.

[22] H. Cheng, S. Chen, et al., Appl. Phys. Lett. 103 (22) (2013) 223102. 
[23] G. Yao, F. Ling, et al., Optoelectronic Devices and Integration. JW3A.38 (2015).

[24] M. Chen, W. Sun, et al., Plasmonics. 11 (2016) 1-7.

[25] L. A. Fal'kovskii, J. Exp. Theor. Phys. 115 (2008) 496-508.

[26] V. Ashkan, and E. Nader, Science. 332 (6035) (2011) 1291-4.

[27] H. Yan, X. Li, B. Chandra, et al., Nat. Nanotechnol. 7 (2012) 330-334.

[28] J. Hao, Q. Ren, Z. An, et al., Phys. Rev. A. 80(2) (2009) 92-92.

[29] Y. J. Chiang, T. J. Yen, Appl. Phys. Lett. 102 (1) (2013) 011129.

[30] G. W. Hanson, IEEE Trans. Antenn. Propag. 56(3) (2008) 747-757.

[31] K. S. Novoselov, A. K. Geim, et al., Science. 306(5696) (2004) 666-9.

[32] K. S. Novoselov, A. K. Geim, et al., Nature. 438(7065) (2005) 197-200.

[33] F. H. L. Minovkoppensich, et al., Nano. Lett. 11 (8) (2011) 3370-3377.

[34] M. Furchi, A. Urich, et al., Nano. Lett. 12 (6) (2012) 2773-2777.

[35] J. Ding, B. Arigong, H. Ren,et al., Plasmonics. 10 (2) (2015) 351-356.

[36] A. Fallahi, J. Perruisseau-Carrier, Phys. Rev. B. 86(19) (2012) 4608-4619.

[37] A. Fardoost, F. G. Vanani, S. A. Amirhosseini, et al., PP(99) (2016) 1-1. 